\title{
Case-level Prediction of Motion Outcomes in Civil Litigation
}

\author{
Devin J. McConnell \\ devin.mcconnell@uconn.edu \\ Department of Computer Science \\ University of Connecticut \\ Storrs, Connecticut, USA \\ Sachin Pandya \\ sachin.pandya@uconn.edu \\ School of Law \\ University of Connecticut \\ Hartford, Connecticut, USA
}

\author{
James Zhu \\ james.zhu@uconn.edu \\ Department of Computer Science \\ University of Connecticut \\ Storrs, Connecticut, USA \\ Derek Aguiar \\ derek.aguiar@uconn.edu \\ Department of Computer Science \\ University of Connecticut \\ Storrs, Connecticut, USA
}

\begin{abstract}
Lawyers regularly predict court outcomes to make strategic decisions, including when, if at all, to sue or settle, what to argue, and how to reduce their clients' liability risk. Yet, lawyer predictions tend to be poorly calibrated and biased, which exacerbate unjustifiable disparities in civil case outcomes. Current machine learning (ML) approaches for predicting court outcomes are typically constrained to final dispositions or are based on features unavailable in real-time during litigation, like judicial opinions. Here, we present the first ML-based methods to support lawyer and client decision making in real-time for motion filings in civil proceedings. Using the State of Connecticut Judicial Branch administrative data and court case documents, we trained six classifiers to predict motion to strike outcomes in tort and vehicular cases between July 1, 2004 and February 18, 2019. Integrating dense word embeddings from complaint documents, which contain information specific to the claims alleged, with the Judicial Branch data improved classification accuracy across all models. Subsequent models defined using a novel attorney case-entropy feature, dense word embeddings using corpus specific TF-IDF weightings, and algorithmic classification rules yielded the best predictor, Adaboost, with a classification accuracy of $64.4 \%$. An analysis of feature importance weights confirmed the usefulness of incorporating attorney case-entropy and natural language features from complaint documents. Since all features used in model training are available during litigation, these methods will help lawyers make better predictions than they otherwise could given disparities in lawyer and client resources. All ML models, training code, and evaluation scripts are available at https://github.com/aguiarlab/motionpredict.
\end{abstract}

\section{CCS CONCEPTS}

- Applied computing $\rightarrow$ Law; • Computing methodologies $\rightarrow$ Machine learning approaches; Natural language processing.

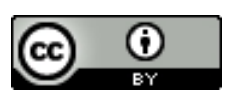

This work is licensed under a Creative Commons Attribution International 4.0 License. ICAIL'21, June 21-25, 2021, São Paulo, Brazil

(C) 2021 Copyright held by the owner/author(s).

ACM ISBN 978-1-4503-8526-8/21/06.

https://doi.org/10.1145/3462757.3466101

\section{ACM Reference Format:}

Devin J. McConnell, James Zhu, Sachin Pandya, and Derek Aguiar. 2021. Case-level Prediction of Motion Outcomes in Civil Litigation. In Eighteenth International Conference for Artificial Intelligence and Law (ICAIL'21), June 21-25, 2021, Sao Paulo, Brazil. ACM, New York, NY, USA, 16 pages. https: //doi.org/10.1145/3462757.3466101

\section{INTRODUCTION}

In the late nineteenth century, American jurist Oliver Wendell Holmes declared that law is "nothing more pretentious" than predictions of what courts in fact do [38]. Lawyers regularly predict what courts will do and, on that basis, make strategic decisions, including what to argue, when, if at all, to sue or prosecute, settle cases, and how to reduce their clients' liability risk. Indeed, because most civil proceedings settle before any trial occurs, litigation outcomes (civil settlements) vary on how attorneys estimate what would happen if the case went to trial and how a trial judge or jury would rule. Yet, despite a literature in litigation decision analysis [13], lawyers' actual predictions tend to be overconfident, not well calibrated [34, 39], and surprisingly vulnerable to bias associated with advocating for a particular view [55].

While lawyers have always made strategic decisions based on what they believed about outcomes in similar cases, the collection of large and diverse data from civil proceedings facilitates a new computational view of legal decision making. This computational perspective of court outcome prediction has been bolstered by the rise of machine learning (ML), which is a branch of artificial intelligence (AI) focused on the development of computational systems that learn from historical data to make predictions or infer patterns in big, noisy, or high-dimensional data sets [28, 74].

Combining ML with collections of dockets, judicial opinions, and court documents presents two significant opportunities. First, ML techniques can increase how accurately lawyers can predict legal outcomes to help support real-time decision making. Computational modeling in law has relied primarily on the text of formal judicial opinions, with or without annotation from domain experts, to classify the outcome reached or the type of legal reasoning used to reach that outcome $[5,35,54,62]$. In some scenarios, ML methods can predict judicial decisions with an accuracy that exceeds legal scholars $[41,65]$. However, court opinions are not available during litigation, and thus this retrospective process stands in contrast to predicting judge or jury decision-making itself $[25,57]$. 
Second, since court outcomes vary with attorney quality and client resources, predictions using ML may reduce the litigation disadvantages faced by the poor, racial minorities, and other vulnerable groups [20]. These problems are exacerbated by overwhelming workloads faced by civil courts and lawyers. Increased workloads contribute to workplace strain, which has detrimental effects on the ability to function effectively [40] and, for lawyers, a decreased perception of ability to uphold the law [12]. However, to ensure widespread acceptability and trustworthiness of algorithmic decisions [60], models must be accurate and explainable to all parties.

Methods for characterizing judicial decisions in previous work have focused on court opinions, ignoring the many important formal procedures that lead to a final judgment. In this work, we present the first client and lawyer support methods that predict court outcomes at the level of individual motions. Motions are formal requests to judges for an official ruling on a contested issue. They can be submitted before, during, or after the trial and can have a significant impact on the final disposition of the lawsuit. For example, motions to strike petition for the removal of all or a subset of the opposing party's pleading. While many court documents are filed over the course of legal proceedings that are relevant to motion outcomes, we focus on complaint documents because (a) they contain the facts alleged and legal claims asserted and (b) are available to all parties when a lawsuit begins and can therefore be used to support decision making.

We present a general overview of computational prediction of litigation outcomes and our contributions in Section 2. Section 3 provides details of our methods, where we describe the court administrative data and legal documents as well as our approach to feature engineering and predictive modelling. We present results on approximately 15 years of Connecticut civil case data in Section 4, followed by a discussion and conclusions in Sections 5 and 6.

\section{BACKGROUND}

Predicting legal outcomes has traditionally been the purview, not only of practicing lawyers, but also researchers of judicial behavior in law, political science, and recently, computer science [37]. Early efforts to computationally model legal decision making focused on representations of rules obtained from case law and legislation [68, $32,64]$. When legal decisions can be modelled as a deterministic process, rule-based AI has achieved considerable success [16, 23] More recently, ML has made a large impact on the research and practice of law in general, and in predictive litigation analysis specifically $[2,3,28,63]$.

Several efforts are focused on extracting standardized data sets to support ML in law. The Supreme Court Database contains over two hundred years of U.S. Supreme Court cases each containing hundreds of variables [70]. The CASELAW4 data set contains 350,000 common law judicial decisions extracted from US State appellate courts [62]. The University of Oxford is constructing a database of 100,000 US court case decisions with features that include the facts of the case, judgements, location, timing, and judicial opinions [24]. These and other similar works [77] provide benchmarks that will accelerate the use of ML in litigation in a similar manner as CIFAR [44] and MNIST [49] for image classification.
The most prevalent features used by ML methods to model court decisions are extracted from legal documents. Legal documents, typically court records, judicial opinions, and legislation, are difficult to model due to their high dimensionality. For instance, modelling documents as bags-of-words, which treats a document as an unordered multiset of words, is commonly assumed in applications like text classification [43] and topic modelling [9]. With this simplifying assumption, the dimension of a document is proportional to the vocabulary size, $|V|$, which is prohibitively large for many ML methods. When the ordering of words in a document is considered, the dimensionality grows exponentially. Therefore, methods typically seek lower dimensional representations of legal documents that preserve relevant structure of the underlying text.

Early legal document representations focused on summary statistics, like word length [8] or other metadata including document complexity [22], publication date, and amendment counts [47]. Knowledge representations of the dyadic citation relationships between documents are typically modelled as citation networks, where vertices correspond to legal documents and a directed edge exists from document $A$ to document $B$ if $A$ cites $B$ [27]. Important features can be extracted from the connectivity structure of legal citation networks, e.g., directed paths can be interpreted as chains of legal precedent or network centrality and in-degree can indicate case importance.

More recently, representations from natural language processing (NLP) have been used to compute richer representations of legal documents. Term frequency-inverse document frequency (TD-IDF) is a statistic computed for a word $w$ and document $d$ :

$$
f_{T}(w, d)=f_{d}(w) \cdot \log \left(\frac{|\mathcal{D}|}{\sum_{d \in \mathcal{D}} \mathbb{1}(w \in d)}\right)
$$

where $d \in \mathcal{D}$ is a document in corpus $\mathcal{D}, f_{d}(w)$ is the frequency of word $w$ in document $d$, and $\mathbb{1}(w \in d)$ is an indicator function that is 1 if $w$ appears in $d$ and 0 otherwise. TD-IDF is often used as a corpus-specific importance weighting for words [80].

State-of-the-art language embedding models have seen recent success by providing lower $d$ dimensional embeddings of words and documents, where $d \approx 10^{2}<<|V|$. These architectures are pretrained on large general corpora and then either applied directly to legal documents or fine-tuned using transfer learning to legal specific applications. The law2vec model is a neural embedding architecture based on word2vec [56] that was pre-trained on a large legal corpus consisting of mostly legislative documents [14]. Taking advantage of recent developments in NLP, some researchers have used transfer learning techniques from pre-trained transformer models [21] to classify U.S. Fourth Amendment cases [35].

Machine learning methods for predicting court outcomes have, thus far, been mostly trained using judicial opinions. One study developed a random forest classifier to predict over 240,000 justice votes and about 28,000 case outcomes for the U.S. Supreme Court from 1816 through 2015 [41]. The method predicted court decisions with $70.2 \%$ accuracy and justice votes with $71.9 \%$ accuracy. By comparison, legal experts at best accurately predicted about $66 \%$ of the outcomes in sixty-eight cases argued in the U.S. Supreme Court's 2002 Term [65]. It is a common practice to use a court's past decisions to predict its future decisions, as was done with data from the European Court of Human Rights [54]. French Supreme 

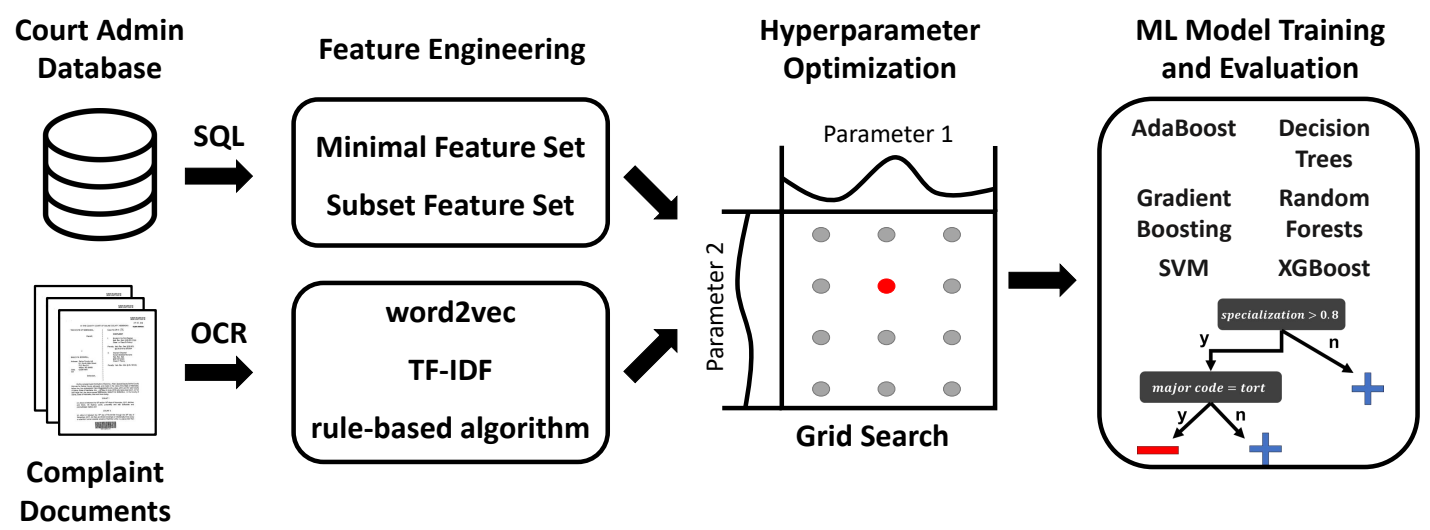

Figure 1: Motion prediction pipeline overview. Two sets of features were computed from the State of Connecticut Judicial Branch court administrative database (minimal and subset, Table 1) and combined with natural language features extracted from complaint documents using word2vec, TF-IDF, and a rule-based algorithm. We optimized the hyperparameters of six ML models using grid search. Here, grid search is described in two dimensions where the circles denote parameter configurations and the curves on each axis denote the marginal classification accuracy. In this example, classification accuracy has higher variability across values of parameter 1, which primarily determines the choice of the best parameter setting (red point). A toy decision tree on the attorney specialization and major code features is shown under our six ML models.

Court decisions have been modelled from historical rulings data using a linear support vector machine (SVM) classifier, assuming a bag-of-words representation for the rulings documents [73].

Importantly, these methods require data from decisions and opinions, which distinguish them from other uses of ML to support real-time litigation support in court cases [25, 57]. Other tools, like MyOpenCourt, provide an AI platform directly to clients for answering legal questions [45]. While these tools do provide real-time support for decision making, the focus is on data mining and learning legal recommendations to support self-represented litigants, not predictive analytics.

\subsection{Our Contributions}

Existing approaches to predicting court case outcomes focus on final judgements of a trial and rely on retrospective court data from appellate or national court decisions, and thus are not amenable for informing motion-level decision making [4, 41, 54, 73]. In this work, we contribute to the field of legal analytics in several ways:

(1) we define new lower dimensional features to support predictive modelling at the case-level;

(2) we develop and benchmark the first computational pipeline to assist lawyer and client decision making through the prediction of motion outcomes in district court data (Figure 1);

(3) we analyze the predictability of motions to strike using both court administrative data and natural language features extracted from complaint documents;

(4) we provide this pipeline (code, trained models, evaluation scripts) freely available and open-source.

\section{METHODS}

Consider $n$ motions filed in civil court whose outcomes are represented as a collection of random variables $\left(y_{1}, \ldots, y_{i}, \ldots, y_{n}\right)^{T}=$ $Y \in\{1, \ldots, k\}^{n}$ where $k$ is the number of distinct judicial rulings.
In this work, we consider $k=2$ where 1 and 2 correspond to motion denied and granted respectively. Let the observed data be $\left(\boldsymbol{x}_{1}, \ldots, \boldsymbol{x}_{i}, \ldots, \boldsymbol{x}_{n}\right)=\boldsymbol{X} \in \mathbb{R}^{n \times p}$, an $n \times p$ matrix of $n$ civil court cases each containing $p$ covariates. Note that $\boldsymbol{X}$ can, in general, contain real, nominal, ordinal, or integer valued variables. Given a training set $(\boldsymbol{X}, \boldsymbol{Y})$, the goal is to build a model that predicts class labels $\overline{\boldsymbol{Y}}_{\text {test }}$ from held-out test data $\boldsymbol{X}_{\text {test }}$ to maximize classification accuracy $\frac{T P+T N}{\left|\boldsymbol{Y}_{\text {test }}\right|}$ where TP and TN are true positives and true negatives respectively.

\subsection{Connecticut Civil Court Data}

The values for $\boldsymbol{X}$ and $\boldsymbol{Y}$ were collected from the State of Connecticut Judicial Branch, which provides access to materials such as public records and court case documents, as well as researcher access to their civil court administrative data [71]. The court administrative data is populated by courthouse staff and stored in a centralized relational database, which was rebuilt locally in mySQL. Court case documents are scanned and made available through the Judicial Branch Law Library API.

We focus on predicting the outcomes for motions to strike. A motion to strike has significant influence on case outcomes and are therefore an important factor in legal decision making. In Connecticut, a motion to strike is a written petition typically from a defendant to a judge to remove part or all of a plaintiff's complaint allegations based on legal insufficiency. While we restrict our attention to civil cases filed in Connecticut, the methods apply more generally to other states as long as minimal docket information and complaint documents are available.

\subsection{Feature Engineering: Court Administrative Data}

Most features in the court administrative data were either not relevant to motion outcomes, had high missingness rates, or were 
categorical variables with a number of levels proportional to the size of the data. Therefore, we developed custom SQL scripts to extract 3 informative features based on domain expertise and low missing data rates $(<0.6 \%$ missingness). In total, we considered four court administrative features: juris number, major code, case location, and attorney specialization (Table 1). The juris number is a unique identifier for the attorney or firm representing the defendant. The major code represents the case type encoded as a Bernoulli variable for tort or vehicular cases. The case location encodes a 15 dimensional categorical variable denoting the Connecticut superior court location for the case.

The attorney specialization is derived based on the entropy of the case type (i.e., major case code) distribution for each attorney. Formally, let the number of different major case codes (e.g. tort or vehicular) associated with an attorney be $m$ and the counts of cases litigated by an attorney in each major case code be $w=$ $\left(w_{1}, \ldots, w_{m}\right)$. Then, we model the case counts $w$ for attorney $a$ as a multinomial distribution with a Dirichlet prior,

$$
\begin{aligned}
w_{a} & \sim \operatorname{Multinomial}\left(\theta_{a}\right) \\
\theta_{a} & \sim \operatorname{Dirichlet}\left(\alpha_{1}, \ldots, \alpha_{m}\right)
\end{aligned}
$$

In this work, we set $\left(\alpha_{1}, \ldots, \alpha_{m}\right)=1$. After observing $w_{a}$, the posterior is a Dirichlet-multinomial distribution

$$
P\left(\theta_{a} \mid w_{a}\right)=\operatorname{Dir}\left(\alpha_{1}+w_{a 1}, \ldots, \alpha_{m}+w_{a m}\right)
$$

We compute the specialization for attorney $a$ and major case code $j$ as the entropy of the posterior expectation:

$$
H\left[E\left[\theta_{a j} \mid w_{a}\right]\right]=-\sum_{j=1}^{m} \frac{w_{a j}+\alpha_{j}}{\sum_{k=1}^{m}\left(w_{a k}+\alpha_{k}\right)} \log \left(\frac{w_{a j}+\alpha_{j}}{\sum_{k=1}^{m}\left(w_{a k}+\alpha_{k}\right)}\right)
$$

where $H$ is Shannon's entropy whose probability vector is the relative frequency of the $j^{\text {th }}$ major case code smoothed by taking the expectation of a Dirichlet-multinomial distribution with prior $\operatorname{Dirichlet}\left(\alpha_{1}, \ldots, \alpha_{m}\right)$; this smoothing reduces the variance of entropy calculations for attorneys with small caseloads. Note, that this generates $m-1$ features.

We consider two feature sets derived from these four court administrative features, minimal and subset (see Table 1).

\begin{tabular}{|l|l|c|}
\hline Feature & Description & S \\
\hline Juris Number & Defendant attorney or firm identifier & \\
\hline Attorney & Case type specialization smoothed by a & \\
Specialization & Dirichlet-multinomial prior & \\
\hline Major Code & Classifies the case type (e.g. tort) & $\checkmark$ \\
\hline Case Location & Superior court location for the case & $\checkmark$ \\
\hline
\end{tabular}

Table 1: Features of the CT Civil Case Administrative Database. The column identified as $S$ denotes whether or not the feature was only included in the subset feature set (and not in the minimal feature set).

\subsection{Feature Engineering: Complaint Documents}

We downloaded 7904 complaint documents associated with each case in our data as PDF files from the State of Connecticut Judicial
Branch website using custom crawling scripts [71]. If a PDF contained text, we used pdftotext (version 0.26.5) to convert the PDF to a text file (see Supplemental Methods for additional details). If a PDF contained an image, we first converted the PDF to a TIFF file using ImageMagick (version 6.9.10-68 Q16) [72]. Then, we converted the TIFF to text using tesseract (version 4.1.1-rc2-20-g01fb) [69]. The tesseract optical character recognition (OCR) engine is based on LSTM neural networks and maintained by Google. It has shown to have high accuracy on machine-written characters and black and white images, both of which categorize complaint documents [75].

3.3.1 Rule-based features for complaint documents. We also consider algorithmically generated natural language features based on a sequential covering rule generating algorithm [1]. A rule $R$ maps a condition (antecedent) to a class (consequent). Here, antecedents are a conjunction of Boolean conditions indicating the presence of a word in a complaint document and the consequent is motion granted or denied. For example, the rule (car $\in D$ ) AND (accident $\in D$ ) AND (negligence $\in D$ ) $\Rightarrow 1$ would map a motion to strike associated with complaint $D$ to denied if the words "car", "accident", and "negligence" are contained within $D$.

The sequential covering algorithm proceeds by learning a rule that maximizes some function. Then, the rule is added to a list and all of the complaint documents covered by this rule are removed from the data. This process is repeated until all documents are removed or sufficient coverage of the data is reached. In this work, we learned the number of rules by cross-validation on the training set. We consider two functions (or criteria) to optimize: a simple criteria and the First Order Inductive Learner (FOIL) criteria.

The function with which the simple sequential covering algorithm optimizes is

$$
\frac{n^{+}+1}{n_{*}+k}
$$

where $n^{+}$is the frequency of a word that appears in the granted (GR) documents, $n_{*}$ is the total number of occurrences of the word across all documents, and $\mathrm{k}$ is the number of classes (here, $k=2$ ). In other words, the simple algorithm greedily adds a term to the antecedent that increases the rule's accuracy the most.

A separate function is the FOIL criteria, which is optimized by the RIPPER algorithm $[17,18]$. The FOIL criteria is less greedy than Equation 1, attempting to balance information gain with document coverage

where

$$
n_{2}^{+}\left(\log _{2} \frac{n_{2}^{+}}{n_{2}^{+}+n_{2}^{-}}-\log _{2} \frac{n_{1}^{+}}{n_{1}^{+}+n_{1}^{-}}\right)
$$

- $n_{1}^{+}\left(n_{1}^{-}\right)$is the number of complaint documents associated with a motion to strike that were granted (denied) that the rule covers;

- $n_{2}^{+}\left(n_{2}^{-}\right)$is the number of complaint documents associated with a motion to strike that are changed to positive (negative) with the addition of a prospective word to the antecedent.

3.3.2 Word embeddings for complaint documents. We considered three architectures to construct complaint document features from neural embeddings: word2vec [56], doc2vec [48], and law2vec [14]. The word2vec model maximizes $\log P\left(w_{O} \mid w_{I}\right)$, or the log probability of a given word $w_{O}$ given an input word $w_{I}$. The doc $2 \mathrm{vec}$ model 
is similar but instead of conditioning on an input word $w_{I}$, it conditions on a vector representing the document. We also consider the law2vec model which was trained on 123,066 documents, including 53,000 UK legislative documents, 62,000 European legislative document, and thousands of other English legislative, U.S. code, and opinion documents. We computed 300 dimensional embeddings for each complaint document using doc2vec and word2vec models that were pre-trained on AP News, Google News, and Wiki articles. The law2vec repository provides pretrained models for 100 and 200 dimensional vectors, thus we selected 200 dimensional vectors. To compute a document representation using word2vec or law2vec, we computed an average word2vec vector weighted by term frequency-inverse document frequency (TF-IDF) [53].

We also produce models trained on a combination of word embedding and rule classifiers. A simple word2vec or simple law2vec TFIDF model computes an average word2vec vector for each document that is weighted by TF-IDF and including only those words identified by the simple rule-based classifier. Likewise, a FOIL word2vec or FOIL law2vec TF-IDF model computes an average word2vec vector for each document that is weighted by TF-IDF and including only those words identified by the FOIL rule-based classifier.

\subsection{Modelling Motion Outcomes}

We considered six classification models to predict motion to strike outcomes: adaBoost [29], decision trees [66], gradient boosting [31], random forests [11], SVM [10, 19], and XGBoost [15]. These methods were selected based on their relatively high explainability [81], applicability in small-data settings, and ability to model mixed data types (e.g. real and categorical-valued variables).

3.4.1 Tree Classifiers. Tree classifiers aim to construct a hierarchy of decision rules that recursively split the data until a leaf is reached that denotes the inferred class label. Formally, the hierarchy is represented as a tree where internal nodes denote a bifurcation of a subset of samples based on maximum information gain splits (although splitting based on other criteria like Gini index is also common). Information gain is the difference between the information entropy of the samples at an internal node in the decision tree and the conditional entropy of the samples split on a feature. Intuitively, after an internal node split, the bifurcation should yield subsets that have higher purity. Since finding an optimal decision tree is NP-hard [36, 46], trees are typically constructed in a greedy fashion with successive maximum entropy gain splits and leaves that define a sample's classification. In this work we build decision tree classifiers [66], which produce a single decision tree, and random forest classifiers [11], which are ensembles of decision trees built from a bootstrap subset of the training data.

3.4.2 Boosting Methods. Boosting is a general ML technique based on building an accurate learner from an ensemble of weak learners [30]. Boosting classifiers learn an ensemble of weak learners (e.g. shallow decision trees) sequentially. In this work, we train Adaboost, gradient boosting trees, and XGBoost classifiers. Adaboost, the first practical boosting algorithm [67], builds new decision trees with successive boosting iterations re-weighting training instances such that newly built decision trees focus more on the samples that were previously misclassified. Gradient boosting trees generalize
Adaboost, optimizing an approximate negative gradient of the binomial deviance loss function [31]. XGBoost, or Extreme Gradient Boosting, is a highly efficient implementation of gradient boosting trees with an adjusted loss function to control the complexity of the decision trees [7]

$$
\begin{aligned}
L_{x g b} & =\sum_{i=1}^{n} L\left(y_{i}, F\left(x_{i}\right)\right)+\sum_{m=1}^{M} \Omega\left(h_{m}\right) \\
\Omega\left(h_{m}\right) & =\gamma T_{m}+\frac{1}{2} \lambda\|s\|^{2}
\end{aligned}
$$

where $L\left(y_{i}, F\left(x_{i}\right)\right)$ is a loss function computed from observed motion outcome $y_{i}$ and computed outcome $F\left(x_{i}\right), h_{m}$ is the $m^{\text {th }}$ weak learning model with $T_{m}$ leaves and $s$ leaf output scores.

3.4.3 Support Vector Machines. Support vector machines classify data by solving a convex optimization problem yielding a hyperplane that optimally separates two classes $[10,19]$. Optimality is measured with respect to the size of the margin between granted and denied motions. They can efficiently compute non-linear decision boundaries with kernel functions, representing similarities in an inner product space.

\section{RESULTS}

The court administrative data from the State of Connecticut Judicial Branch contained 916,805 observations from 184,125 unique cases. First, we extracted 7904 motions to strike filed by a defendant against a plaintiff between July 1, 2004 to February 18, 2019. In the results that follow, we focus on predicting the outcome of a motion to strike in civil tort and vehicular cases based on court administrative data and complaint documents.

Classifier performance can greatly depend on model selection [26]. For example, when using default parameters, the performance of XGBoost is significantly worse than models with optimized hyperparameters [7]. For model selection, we performed a grid search for each method, with 10 fold cross validation on $70 \%$ of the data used

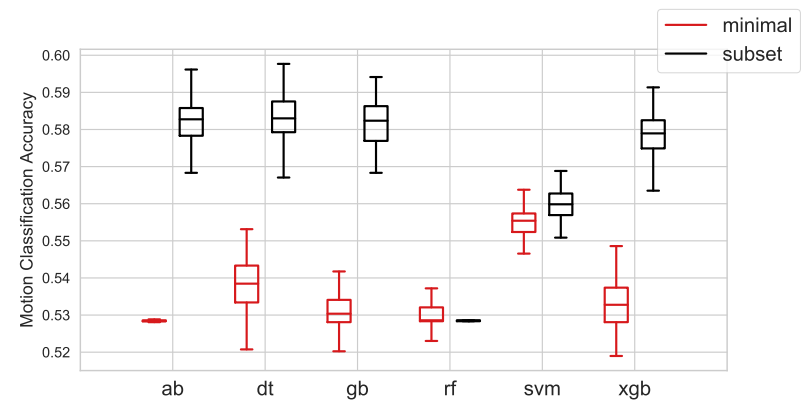

Figure 2: Performance comparison by feature set using court administrative database features. We compared the motion to strike classification accuracy on minimal and subset feature sets for six ML models (adaBoost (ab), decision trees (dt), gradient boosting (gb), random forests (rf), support vector machines (svm), and XGBoost (xgb)). Box plots show the distribution of 100 bootstrapped samples with Tukey whiskers (median \pm 1.5 times interquartile range). 


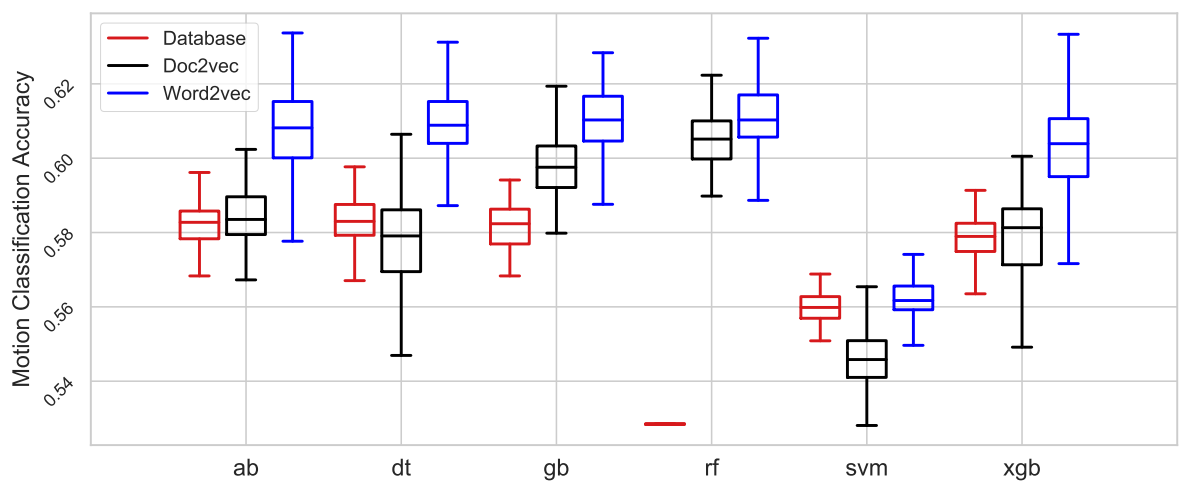

Figure 3: Predicting motion to strike outcomes across court administrative data and complaint documents. Distinct classifiers were trained on court administrative data and dense features computed from complaint documents. Document embedding features based on doc2vec [48] (black) and word2vec [56, 53] (blue) largely improved the classification accuracy of motion outcomes versus court administrative database features alone (red) for six classifiers [61]: AdaBoost (ab), decision trees (dt), gradient boosting (gb), random forests (rf), support vector machines (svm), Xgboost (xgb). Box plots are drawn with Tukey whiskers (median \pm 1.5 times interquartile range).

for training and validation. To estimate variability in classification accuracy, we computed 100 bootstrapped samples for each model selected from our grid search.

First, we evaluated the minimal and subset feature sets (Table 1) associated with legal cases to determine feature relevancy for motion outcome prediction. We varied the feature composition for the court administrative data and compared classifier accuracy (Fig. 2). Adding in the case location and major code features improved median accuracy for all methods besides random forests. Overall, decisions trees exhibited the highest motion to strike classification accuracy (mean: 0.583 , median: 0.583 ) with slightly higher performance than boosting methods. Given these results, we primarily focus our analysis on the subset featurization.

To assist interpretation of model performance, we compared our ML models with a naive baseline. The naive classifier predicts motion outcomes using the empirical frequency of the training set; with $52 \%$ of the motions being granted, we observed a naive baseline accuracy of 0.501 . During model selection, we observed a maximum classification accuracy of 0.644 using Adaboost with dense word embeddings, corpus specific TF-IDF weightings, and FOIL algorithmic rules. This same model had a mean accuracy of 0.605 over 100 bootstraps. However, the highest mean accuracy score from the same group of features was found in decision tree classifiers with 0.606

Next, we evaluated whether court administrative data alone was sufficient for learning accurate motion to strike classification models. Using only database features, all methods produced classification accuracies less than 0.60 (Fig. 3). Subsequently, we evaluated these same methods, but including dense natural language features extracted from complaint documents. While concatenating doc2vec features to the database feature vectors improved classification accuracy for most methods, a more careful model defined over word2vec features using corpus specific weighting (TF-IDF) improved classification accuracy for all models. These results highlight the utility of incorporating natural language features in the prediction of motion outcomes.

Interestingly, the difference between minimal and subset featurization was diminished when including complaint document features into the model. We observed this behavior when considering both doc2vec (Supp. Fig. 1) and word2vec (Supp. Fig. 2) features, although word2vec features continued to yield better performing models. These findings suggest that complaint document embeddings effectively capture major code and case location features.

We next investigated feature significance for motion to strike outcome prediction for decision trees built on word2vec features. We chose word2vec features because models built on word2vec embeddings produced more accurate results than database-only or database and doc2vec features; we selected decisions trees since these are the most explainable of the six models tested with similar performance to the boosting models. We found that features derived from the complaint documents were universally important across all decision tree classifiers (Fig. 4). The case type (Major Code) was also an important feature for a subset of models, as was the attorney specialization (entropy).

Next, we quantified whether tort or vehicular cases had subtle differences that made prediction easier by stratifying classification accuracy by case type in our best performing feature configuration (database features, FOIL and TF-IDF weighted word2vec). All methods, excluding SVM, predicted motion to strike outcomes in vehicular cases with a significantly higher accuracy than tort cases (one-way paired $t$-test, $p \leq 2.2 \times 10^{-16}$ ) (Fig. 5). This is likely due to inherent properties of vehicular cases and not class imbalance since vehicular cases encompassed approximately $47 \%$ of the total cases.

Lastly, we investigated if legal domain-specific word2vec models would improve classifier performance compared to word2vec 


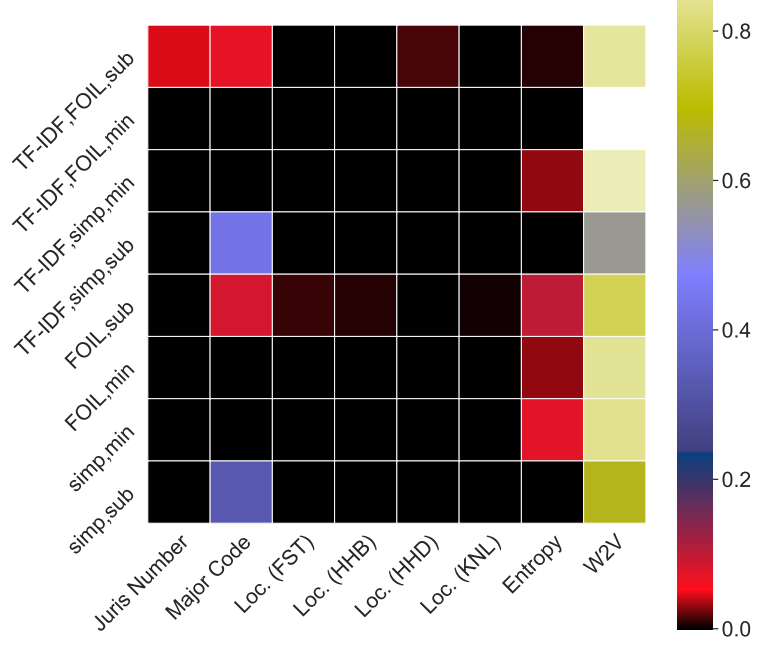

Figure 4: Decision tree feature importance for word2vec models. The vertical axis denotes the model configuration from TF-IDF weighted, simple (simp) or FOIL algorithmic rules, and minimum ( $\mathrm{min}$ ) or subset (sub) feature sets. The $\mathrm{x}$-axis gives features with at least one model yielding a nonzero importance score. The case major code, attorney specialization (entropy), and word2vec (W2V) features exhibited the highest average feature importance across models. The word2vec feature importance weights were summed across all word2vec features.

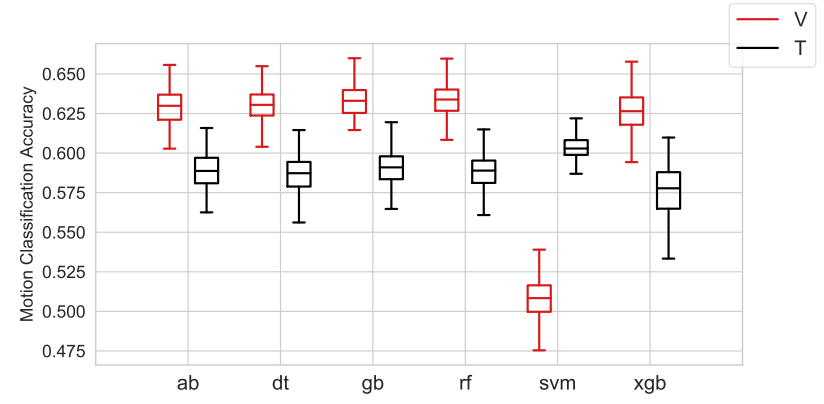

Figure 5: Classifier accuracy by case type. We stratified classifier performance on motion to strike prediction by vehicular or tort case types (major code). Here, we include classifiers trained on court administrative and word2vec features. Box plots show the distribution of 100 bootstrapped samples with Tukey whiskers (median \pm 1.5 times interquartile range).

trained on general corpora [42]. Overall, we did not find that

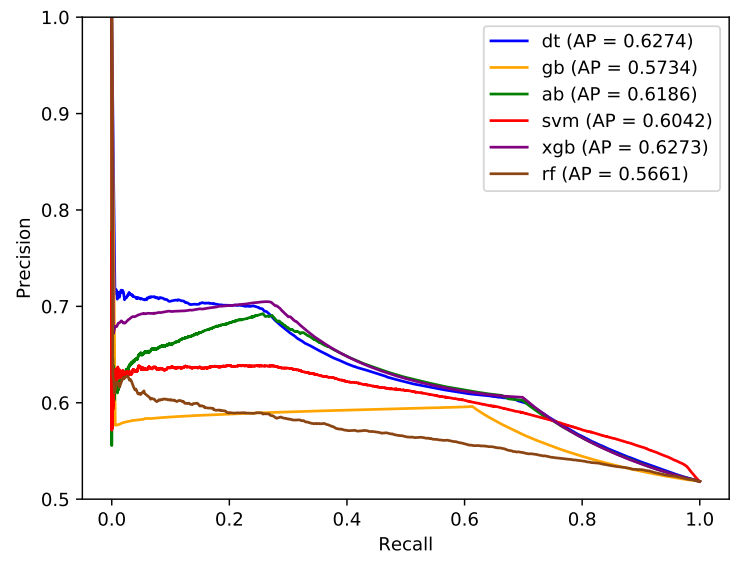

Figure 6: Precision-Recall curves for law2vec classifier on subset and TF-IDF weighted simple features. We trained and tested with 100 bootstrapped samples the law2vec model using TF-IDF weighted simple and subset features. Precision and recall were computed for several thresholds for six ML models (adaBoost (ab), decision trees (dt), gradient boosting (gb), random forests (rf), support vector machines (svm), and XGBoost $(\mathrm{xgb}))$. Average precision (AP) for each method is given in the legend.

law2vec improved classifier performance. Using court administrative data, FOIL, and TF-IDF weighted features, word2vec classifier median performance was greater than or approximately equal across all methods besides XGBoost (Supp. Fig. 3); the behavior was similar when removing FOIL features (Supp. Fig. 4). While tree and boosting classifiers outperformed our SVM model overall, when we compared the performance of each classifier by varying the classification threshold, all methods had similar average precision when including TF-IDF weighted features from the simple (Fig. 6) and FOIL (Supp. Fig. 5) rule based classifiers or when including (Supp. Fig. 6) and excluding (Supp. Fig. 7) FOIL features without TF-IDF weighting. However, SVMs have noticeably higher average precision than their classification accuracy would suggest, providing strong justification for SVMs when multiple classification thresholds are considered.

\section{DISCUSSION}

In this work we focused on motions to strike, but the prediction of other high impact motions may be of interest to lawyers and their clients. For example, outcomes of interest may include the file dates of a disposition, the cost or activity associated with the discovery period (which is typically a function of complexity of motions during discovery), or motions for default judgement which can happen when a party does not respond to a court filing. A motion for summary judgement is another high-impact motion which occurs with high frequency; this motion is a written petition which argues that, based on the evidence gathered thus far, no reasonable jury could find that the plaintiff has proved one or 
more genuinely-disputed facts that matter to one or more of the plaintiff's legal claim. However, careful consideration must be taken when deciding which features or court documents are relevant. For example, a motion for summary judgement depends on the quality of the evidence collected by the parties thus far, which may not be well captured by the text of court documents.

Our comparisons between doc2vec and law2vec suggested there are potential benefits to incorporating neural embeddings from models trained specifically on legal corpora. In modern transformer architectures, domain specific pre-trained models, e.g. BioBERT [51], have been shown to outperform transfer learning fine-tuning approaches from general corpora like Wikipedia. While some of these models are in the legal domain, e.g. patentBERT [50], no such model exists for court documents. However, one significant challenge to applying such models in the legal domain is that the memory requirements for transformers scale quadratically with sequence length, prohibiting them from being applied directly to the longer texts that are common in complaints and judicial opinions [6]. Recent work on extending the range of transformers provides some evidence that this issue will be addressed $[79,76]$.

A limitation of the data in our analysis is that courts may occasionally grant a motion to strike in part. This can occur, for example, if two or more legal claims levied against a defendant were formally challenged by a motion to strike. The court may grant the motion to strike with respect to a single legal claim and deny it for others. In these cases, the motion to strike order code in the Law Library data is noisy since there is only a single value that is provided. Furthermore, the procedure with which courts interpret this order code is heterogeneous. Some courts interpret a granted motion as any motion granted in part or in full; other courts only use the granted order code when the motion is granted in full. Addressing this issue either requires reforming and unifying the data processing procedures across Connecticut courts or developing methods to parse out distinct legal claims from complaint documents and then matching them to judicial order documents.

The relevant features for the motion prediction problem can also likely be improved. For example, an attorney is defined based on their juris number and a derived attorney specialization feature. There are other features that are likely relevant for predicting motion outcomes, e.g., attorney experience, record, or case load. Similar feature engineering can be implemented for judges. Case location and other high dimensional categorical features can be one-hot encoded, but may also benefit from a descriptive, lower dimensional set of features based on, e.g., court culture.

With the rise of $M L$ in the legal domain, governments across the globe are placing new emphases on ensuring AI-assisted decision making is done in an ethical, transparent, and nondiscriminatory manner. The European Commission for the Efficiency of Justice adopted 5 principles in the European Ethical Charter on the use of AI in judicial systems [82]. These principles guarantee that judicial AI is compatible with fundamental rights, nondiscriminatory, transparent, impartial, fair, and explainable. In the U.S., the National Center for State Courts has identified data transparency and investigating how AI transforms judicial processes as national priorities [59].

Fundamental to upholding these ideals is developing methods that are interpretable. Interpretability provides a mechanism to ensure ethical and legal compliance in situations where the ethics or legal implications are ill-defined or cannot be mathematically modelled. Model-based interpretability focuses on restricting the set of models such that a trained model directly informs relationships among model variables [58]. The methods developed in this work were selected, in part, due to high model-based interpretability (e.g. decision trees), but, incorporating causal reasoning and explicit Bayesian modelling of relevant variables in the judicial decision making process would only increase explainability, interpretability, and ultimately, trustworthiness of the model. One possibility is to consider the recent work in causal frameworks for decision trees [33, $52,78]$.

\section{CONCLUSIONS}

By developing ML workflows with feature engineering rooted in legal domain expertise, we developed methods to help researchers better understand the predictability of trial motions and practitioners the ability to make more informed decisions. We developed and benchmarked the first ML methods to predict motion outcomes using only data that is available to all parties at trial. Our work demonstrated that motion to strike outcomes are predictable with high accuracy when new features like attorney specialization are combined with complaint document embeddings.

We expect these methods will be a valuable resource for lawyers and their clients by enabling the estimation of case strength. For example, our methods can be used to predict if the case will survive a motion to strike after a complaint document is filed. Based on predicted motion outcomes, both parties can make more informed settlement decisions and lawyers representing the plaintiff can revise the language in their complaint documents. Fitted ML models, training code, and benchmarking code can be accessed at https://github.com/aguiarlab/motionpredict.

\section{REFERENCES}

[1] Charu C Aggarwal. 2018. Machine learning for text. Springer.

[2] Sharan Agrawal et al. 2017. Affirm or reverse? using machine learning to help judges write opinions. NBER Working Paper, 29.

[3] Benjamin Alarie et al. 2016. Using machine learning to predict outcomes in tax law. Can. Bus. Lf, 58, 231.

[4] Nikolaos Aletras et al. 2016. Predicting judicial decisions of the european court of human rights: a natural language processing perspective. Peerf Computer Science, 2, e93.

[5] Katie Atkinson et al. 2020. Explanation in AI and law: past, present and future. Artificial Intelligence, 103387.

[6] Iz Beltagy et al. 2020. Longformer: the long-document transformer. arXiv preprint arXiv:2004.05150.

[7] Candice Bentejac et al. 2020. A comparative analysis of gradient boosting algorithms. Artificial Intelligence Review, 131.

[8] Ryan C Black and James F Spriggs. 2008. An empirical analysis of the length of US Supreme Court opinions. Hous. L. Rev., 45, 621.

[9] David M Blei et al. 2003. Latent dirichlet allocation. the fournal of Machine Learning Research, 3, 993-1022. 
[10] Bernhard E Boser et al. 1992. A training algorithm for optimal margin classifiers. In Proceedings of the fifth annual workshop on Computational learning theory, 144-152.

[11] Leo Breiman. 2001. Random forests. Machine learning, 45, 1, 5-32.

[12] Shelagh MR Campbell. 2017. Exercising discretion in the context of dependent employment: assessing the impact of workload on the rule of law. Legal Studies, 37, 2, 305-323.

[13] John Celona. 2016. Winning at Litigation through Decision Analysis: Creating and Executing Winning Strategies in any Litigation or Dispute. Springer Series in Operations Research and Financial Engineering. Springer.

[14] Ilias Chalkidis. 2018. Law2Vec: Legal Word Embeddings. (2018). https://archive.org/details/Law2Vec.

[15] Tianqi Chen and Carlos Guestrin. 2016. Xgboost: a scalable tree boosting system. In Proceedings of the 22nd acm sigkdd international conference on knowledge discovery and data mining, 785-794.

[16] Cary Coglianese. 2004. E-rulemaking: information technology and the regulatory process. Admin. L. Rev., 56, 353.

[17] William W Cohen et al. 1996. Learning rules that classify e-mail. In AAAI spring symposium on machine learning in information access. Volume 18. Stanford, CA, 25.

[18] William W Cohen and Yoram Singer. 1999. Context-sensitive learning methods for text categorization. ACM Transactions on Information Systems (TOIS), 17, 2, 141-173.

[19] Corinna Cortes and Vladimir Vapnik. 1995. Support-vector networks. Machine learning, 20, 3, 273-297.

[20] Lindsey Devers. 2011. Plea and charge bargaining. Research summary for Bureau of Justice Assistance, U.S. Department of fustice, 1.

[21] Jacob Devlin et al. 2018. Bert: pre-training of deep bidirectional transformers for language understanding. arXiv preprint arXiv:1810.04805.

[22] Michael Evans et al. 2007. Recounting the courts? applying automated content analysis to enhance empirical legal research. Journal of Empirical Legal Studies, 4, 4, 1007-1039.

[23] Frank Fagan and Saul Levmore. 2019. The impact of artificial intelligence on rules, standards, and judicial discretion. $S$. Cal. L. Rev., 93, 1.

[24] Felix Steffek. 2021. Law and Autonomous Systems Series: Paving the Way for Legal Artificial Intelligence - A Common Dataset for Case Outcome Predictions. University of Oxford. (2021). https://www.law.ox.ac.uk/business-law-blog/blog/ 2018 / 05 / law - and - autonomous - systems - series - paving way-legal-artificial.

[25] Norman Fenton et al. 2016. Bayes and the law. Annual Review of Statistics and Its Application, 3, 1, 51-77. https://doi.org/ 10.1146/annurev-statistics-041715-033428.

[26] Matthias Feurer and Frank Hutter. 2019. Hyperparameter optimization. In Automated Machine Learning. Springer, Cham, 3-33.

[27] James H Fowler et al. 2007. Network analysis and the law: measuring the legal importance of precedents at the us supreme court. Political Analysis, 324-346.

[28] Jens Frankenreiter and Michael A. Livermore. 2020. Computational methods in legal analysis. Annual Review of Law and
Social Science, 16, 1, 39-57. https://doi.org/10.1146/annurevlawsocsci-052720-121843.

[29] Yoav Freund and Robert E Schapire. 1997. A decision-theoretic generalization of on-line learning and an application to boosting. Journal of computer and system sciences, 55, 1, 119139.

[30] Yoav Freund et al. 1999. A short introduction to boosting. Journal-Japanese Society For Artificial Intelligence, 14, 771$780,1612$.

[31] Jerome H Friedman. 2002. Stochastic gradient boosting. Computational statistics \& data analysis, 38, 4, 367-378.

[32] Anne von der Lieth Gardner. 1984. Artificial intelligence approach to legal reasoning. Technical report. Stanford Univ.

[33] Tim Genewein et al. 2020. Algorithms for Causal Reasoning in Probability Trees. en. arXiv:2010.12237 [cs], (November 2020). arXiv: 2010.12237. Retrieved 12/10/2020 from http: //arxiv.org/abs/2010.12237.

[34] Jane Goodman-Delahunty et al. 2010. Insightful or wishful: lawyers' ability to predict case outcomes. Psychology, Public Policy, and Law, 16, 2, 133-157.

[35] Evan Gretok et al. 2020. Transformers for classifying fourth amendment elements and factors tests. In Legal Knowledge and Information Systems: FURIX 2020: The Thirty-third Annual Conference, Brno, Czech Republic, December 9-11, 2020. Volume 334. IOS Press, 63-72.

[36] Thomas Hancock et al. 1996. Lower bounds on learning decision lists and trees. Information and Computation, 126, 2 , 114-122.

[37] Allison P. Harris and Maya Sen. 2019. Bias and judging. Annual Review of Political Science, 22, 1, 241-259. https://doi. org/10.1146/annurev-polisci-051617-090650.

[38] Oliver Wendell Holmes. 1897. The path of the law. Harvard Law Review, 10, 8, 457-478.

[39] Jonas Jacobson et al. 2011. Predicting civil jury verdicts: how attorneys use (and misuse) a second opinion. fournal of Empirical Legal Studies, 8, S1, 99-119. http://dx.doi.org/10.1111/ j.1740-1461.2011.01229.x.

[40] Robert A Karasek Jr. 1979. Job demands, job decision latitude, and mental strain: implications for job redesign. Administrative science quarterly, 285-308.

[41] Daniel Martin Katz et al. 2017. A general approach for predicting the behavior of the Supreme Court of the United States. PLOS ONE, 12, 4, (April 2017), 1-18. https://doi.org/ 10.1371/journal.pone.0174698.

[42] Nari Kim and Hyoung Joong Kim. 2017. A study on the law2vec model for searching related law. Fournal of Digital Contents Society, 18, 7, 1419-1425.

[43] Sang-Bum Kim et al. 2006. Some effective techniques for naive Bayes text classification. IEEE transactions on knowledge and data engineering, 18, 11, 1457-1466.

[44] Alex Krizhevsky, Geoffrey Hinton, et al. 2009. Learning multiple layers of features from tiny images.

[45] Jason T Lam et al. 2020. The gap between deep learning and law: predicting employment notice. NLLP KDD, 7, 10.

[46] Hyafil Laurent and Ronald L Rivest. 1976. Constructing optimal binary decision trees is NP-complete. Information processing letters, 5, 1, 15-17. 
[47] David S Law and David Zaring. 2009. Law Versus Ideology: The Supreme Court and the Use of Legislative History. Wm. \& Mary L. Rev., 51, 1653.

[48] Quoc Le and Tomas Mikolov. 2014. Distributed representations of sentences and documents. In International conference on machine learning, 1188-1196.

[49] Yann LeCun and Corinna Cortes. 2010. MNIST handwritten digit database. http://yann.lecun.com/exdb/mnist/.

[50] Jieh-Sheng Lee and Jieh Hsiang. 2019. Patentbert: patent classification with fine-tuning a pre-trained bert model. arXiv preprint arXiv:1906.02124.

[51] Jinhyuk Lee et al. 2020. Biobert: a pre-trained biomedical language representation model for biomedical text mining. Bioinformatics, 36, 4, 1234-1240.

[52] Jiuyong Li et al. 2016. Causal decision trees. IEEE Transactions on Knowledge and Data Engineering, 29, 2, 257-271.

[53] Joseph Lilleberg et al. 2015. Support vector machines and word2vec for text classification with semantic features. In 2015 IEEE 14th International Conference on Cognitive Informatics \& Cognitive Computing (ICCI* CC). IEEE, 136-140.

[54] Masha Medvedeva et al. 2020. Using machine learning to predict decisions of the European Court of Human Rights. Artificial Intelligence and Law, 28, 2, 237-266.

[55] David E. Melnikoff and Nina Strohminger. 2020. The automatic influence of advocacy on lawyers and novices. Nature Human Behaviour, (September 7, 2020), 1-7.

[56] Tomas Mikolov et al. 2013. Efficient estimation of word representations in vector space. (2013). http://arxiv.org/abs/ 1301.3781.

[57] Jane Mitchell et al. 2020. Machine learning for determining accurate outcomes in criminal trials. Law, Probability and Risk, 19, 1, (March 2020), 43-65.

[58] W James Murdoch et al. 2019. Definitions, methods, and applications in interpretable machine learning. Proceedings of the National Academy of Sciences, 116, 44, 22071-22080.

[59] National Center for State Courts. 2021. Joint technology committee priority topics. Accessed on 2021-03-01. (2021). https: //www.ncsc.org/about-us/committees/joint-technologycommittee/priority-topics-old-page.

[60] Patrick W Nutter. 2018. Machine learning evidence: admissibility and weight. U. Pa. F. Const. L., 21, 919.

[61] F. Pedregosa et al. 2011. Scikit-learn: machine learning in Python. Fournal of Machine Learning Research, 12, 2825-2830.

[62] Alina Petrova et al. 2020. Extracting Outcomes from Appellate Decisions in US State Courts. In Legal Knowledge and Information Systems: FURIX 2020: The Thirty-third Annual Conference, Brno, Czech Republic, December 9-11, 2020. Volume 334. IOS Press, 133-142.

[63] Arti K Rai. 2018. Machine learning at the patent office: lessons for patents and administrative law. Iowa L. Rev., 104, 2617.

[64] Edwina L Rissland. 1990. Artificial intelligence and law: stepping stones to a model of legal reasoning. The Yale Law Journal, 99, 8, 1957-1981.

[65] Theodore W Ruger et al. 2004. The supreme court forecasting project: legal and political science approaches to predicting supreme court decision making. Columbia Law Review, 11501210.
[66] S. R. Safavian and D. Landgrebe. 1991. A survey of decision tree classifier methodology. IEEE Transactions on Systems, Man, and Cybernetics, 21, 3, 660-674.

[67] Robert E Schapire. 2013. Explaining adaboost. In Empirical inference. Springer, 37-52.

[68] Marek J. Sergot et al. 1986. The British Nationality Act as a logic program. Communications of the ACM, 29, 5, 370-386.

[69] Ray Smith. 2007. An overview of the tesseract ocr engine. In Ninth international conference on document analysis and recognition (ICDAR 2007). Volume 2. IEEE, 629-633.

[70] Harold Spaeth et al. 2014. Supreme court database code book. (2014).

[71] State of Connecticut Judicial Branch. 2021. Public Records Online. Accessed on 2021-01-01. (2021). https://jud.ct.gov/ lawlib/publicrecords.htm.

[72] Michael Still. 2006. The definitive guide to ImageMagick. Apress.

[73] Octavia-Maria Şulea et al. 2017. Predicting the law area and decisions of French Supreme Court cases. In Proceedings of the International Conference Recent Advances in Natural Language Processing, RANLP 2017. INCOMA Ltd., Varna, Bulgaria, (September 2017), 716-722. https://doi.org/10.26615/ 978-954-452-049-6_092.

[74] Harry Surden. 2014. Machine learning and law. Wash. L. Rev., $89,87$.

[75] Ahmad P Tafti et al. 2016. OCR as a service: an experimental evaluation of Google Docs OCR, Tesseract, ABBYY FineReader, and Transym. In International Symposium on Visual Computing. Springer, 735-746.

[76] Yi Tay et al. 2020. Long Range Arena: A Benchmark for Efficient Transformers. en. arXiv:2011.04006 [cs], (November 2020). arXiv: 2011.04006. Retrieved 12/12/2020 from http: //arxiv.org/abs/2011.04006.

[77] Thomas Vacek et al. 2019. Litigation Analytics: Case outcomes extracted from US federal court dockets. In Proceedings of the Natural Legal Language Processing Workshop 2019, 45-54.

[78] Stefan Wager and Susan Athey. 2018. Estimation and inference of heterogeneous treatment effects using random forests. Fournal of the American Statistical Association, 113, 523, 1228-1242.

[79] Sinong Wang et al. 2020. Linformer: Self-Attention with Linear Complexity. en. arXiv:2006.04768 [cs, stat], (June 2020). arXiv: 2006.04768. Retrieved 12/12/2020 from http://arxiv. org/abs/2006.04768.

[80] Ho Chung Wu et al. 2008. Interpreting tf-idf term weights as making relevance decisions. ACM Transactions on Information Systems (TOIS), 26, 3, 1-37.

[81] Feiyu Xu et al. 2019. Explainable ai: a brief survey on history, research areas, approaches and challenges. In CCF international conference on natural language processing and Chinese computing. Springer, 563-574.

[82] Irina Moroianu Zlatescu and Petru Emanuel Zlatescu. 2019. Implementation of the European ethical charter on the use of artificial intelligence in judicial systems and their environment. Current Issues of the EU Political-Legal Space, 237. 